
СПОНДИЛИТОМ (БОЛЕЗНЬЮ БЕХТЕРЕВА) И ПСОРИАТИЧЕСКИМ АРТРИТОМ ПО РЕЗУЛЬТАТАМ ДЕСЯТИЛЕТНЕГО ПРОСПЕКТИВНОГО НАБЛЮДЕНИЯ (ИССЛЕДОВАНИЕ ПРОГРЕСС)

\author{
И.З. Гайдукова, А.П. Ребров \\ ФГБОУ ВО «Саратовский государственный медицинский университет им. В.И. Разумовского» Минздрава России; \\ Россия, 410012 Саратов, ул. Большая Казачья, 112
}

Контакты: Инна Зурабиевна Гайдукова иbp1976@list.ru

Цель исследования - оценка частоты появления новых случаев ишемической болезни сердиа (ИБС) у больных анкилозирующим спондилитом (AC) и псориатическим артритом (ПсА) без манифестных сердечно-сосудистых заболеваний.

Материалы и методы. Выполнен анализ данных 10-летнего проспективного наблюдения за пациентами с АC ( $n=278)$, псориатическим артритом $(n=85)$ и здоровыми лицами $(n=150)$ без сердечно-сосудистых заболеваний. Лица всех групп сопоставимы по факторам сердечно-сосудистого риска. Учитывали документально подтвержденные кардиологом новые случаи ИБС за 10 лет. Результаты. Новые случаи ИБС за 10 лет были зафиксированы у 64 из 278 пациентов с АС и у 16 из 150 лии группы сравнения ( $p=0,0017)$. Сравнение частоты появления ИБС с применением log-rank Mantel-Cox test u log-rank test for trend noказало значимость различий между частотой появления ИБС улии, без спондилоартрита (CnA), улиц с АС и ПсА (p <0,0001). Риск развития ИБС у больных ПсА превосходил риск развития ИБС у лии контроля: отношение рисков (RR) составил 4,16 (95 \% доверительный интервал (ДИ) 2,36-7,33), RR 6,1 (95 \% ДИ 3,05-12,44) (р <0,05). RR развития инфаркта миокарда относительно здоровых лии был повышен как у больных $A C$ (RR 4,98; 95 \% ДИ 1,54-6, 12), так и у больных ПсА (RR 5,2; 95 \% ДИ 2,4-7,8). Риск развития стенокардии при АС не превышал показатели здоровых лии $(p>0,05)$.

Заключение. Риск развития стенокардии напряжения у больных с АС не превышает аналогичный риск у лии без СпА. При этом пациенты с АС имеют бо́льший риск развития инфаркта миокарда, чем лица без СпА. Лица с ПсА имеют бо́льший риск развития ИБС как по сравнению со здоровыми лицами, так и по сравнению с лицами, страдающими АС.

Ключевые слова: ишемическая болезнь сердиа, инфаркт миокарда, стенокардия, артериальная гипертензия, сердечно-сосудистый риск, спондилоартрит, анкилозирующий спондилит, псориатический артрит, С-реактивный белок, нестероидные противовоспалительные препараты, гипотензивная терапия

DOI: $10.17650 / 1818-8338-2016-10-3-26-31$

\title{
THE RISK OF CORONARY ARTERY DISEASE DEVELOPMENT IN PATIENTS WITH ANKYLOSING SPONDYLITIS (BECHTEREW'S DISEASE) AND PSORIATIC ARTHRITIS: A 10-YEAR PROSPECTIVE FOLLOW-UP STUDY
}

\section{I.Z. Gaidukova, A.P. Rebrov}

V.I. Razumovskiy Saratov State Medical University, Ministry of Health of Russia; 112 Bol'shaya Kazach'ya St., Saratov 410012, Russia

Objective: assessment of coronary artery disease (CAD) incidence among patients with ankylosing spondylitis (AS) and psoriatic arthritis (PSA) without manifestation of cardiovascular diseases.

Materials and methods. We analyzed the data of 10-year prospective follow-up of the patient with $A S(n=278)$, psoriatic arthritis $(n=85)$ and healthy controls $(n=150)$ without any cardiovascular diseases. All groups were comparable in regard to cardiovascular risk factors. During these 10 years all new cases of CAD (verified by cardiologist) in the study population were tracked.

Results. New cases of CAD were fixed in 64 out of 278 patietns with AS and in 16 out of 150 controls ( $p=0.0017)$. Using log-rank MantelCox test and logrank test for trend we demonstrated statistically significant differences in CAD incidence between patients without spondyloarthritis $(S p A)$ and patients with $A S$ and $P_{S A}(p<0,0001)$. The risk of CAD development was higher in PSA group than in the control group; relative risk was 4.16(95\% confidence interval (CI) 2.36-7.33), RR 6.1 (95\% CI 3.05-12.44) $(p<0.05)$. Increased risk of myocardial infarction was observed both in patients with $A S$ (RR 4.98; $95 \%$ CI 1.54-6.12) and patients with PSA (RR 5.2; $95 \%$ CI 2.4-7.8) comparing to healthy controls. There was no significant difference between the $A S$-group and the control group in terms of risk of stenocardia development $(p>0.05)$.

Conclusion. The risk of exertional stenocardia in patients with AS was not higher than that in individuals without SpA. However, patients with AS have higher risk of myocardial infarction than those without SpA. PsA patients have increased risk of CAD development comparing to healthy controls and individuals with $A S$. 
Key words: coronary artery disease, myocardial infarction, stenocardia, arterial hypertension, cardiovascular risk, spondyloarthritis, ankylosing spondylitis, psoriatic arthritis, C-reactive protein, nonsteroidal anti-inflammatory drugs, antihypertensive therapy

\section{Введение}

Сердечно-сосудистые события являются лидирующей причиной смерти больных с анкилозирующим спондилитом (АС) и псориатическим артритом (ПсА) [1-4]. Несмотря на появление в 2015 г. Европейских рекомендаций по мониторингу сердечно-сосудистых заболеваний при спондилоартритах (СпА) [5], многие вопросы, касающиеся патологии сердечно-сосудистой системы при АС и ПсА, нуждаются в уточнении. Так, у больных СпА недостаточно изучена структура сердечно-сосудистой заболеваемости и смертности. Имеющиеся данные противоречивы и не описывают закономерности развития поражения сердечно-сосудистой системы при СпА, отличающие их от общей популяции. В исследовании S. Brophy и соавт. (2012) [6] продемонстрировано отсутствие увеличения частоты фатальных и нефатальных инфарктов миокарда и инфарктов мозга у больных AC, а в исследованиях S. Han и соавт. (2006) и С. Haroon и соавт. (2015) показано увеличение риска инфаркта миокарда при АС [2, 3]. Наше собственное исследование (И.З. Гайдукова и соавт., 2015 г.) не показало повышения сердечно-сосудистой смертности при АС, но продемонстрировало ее повышение у больных ПсА [7]. Для пациентов со СпА нет четких данных о встречаемости сердечно-сосудистых рисков [8-11]. Таким образом, изучение структуры сердечнососудистой заболеваемости при СпА остается актуальной проблемой.

Цель настоящей работы - оценка частоты появления новых случаев ишемической болезни сердца у больных АС и ПсА манифестных сердечно-сосудистых заболеваний.

\section{Матершалы и методы}

\section{Дизайн работы}

Настоящая работа выполнена на основании данных, полученных в ходе проспективного когортного одноцентрового исследования по изучению функционального статуса, активности и сопутствующей патологии (включая сердечно-сосудистую заболеваемость) пациентов со СпА, инициированного в 2004 г. на базе ревматологического отделения ГУЗ «Областная клиническая больница» (г. Саратов), в последующем зарегистрированного как «ПРОГрамма монитоРинга активности и функционального статуса пациЕнтов со Спондилоартритами в Саратовской области (ПРОГРЕСС) проспективное когортное одноцентровое исследование» (регистрация на сайте www.citis.ru № 01201376830 от 09.12.2013). В период с 2004 по 2015 г. в исследование были включены 676 пациентов со СпА, госпитализированных или консультировавшихся амбулаторно на базе ревматологического отделения, не имевших манифестной сердечно-сосудистой патологии на момент включения, кроме контролируемой артериальной гипертензии I-II стадии. За 10 лет наблюдения 313 пациентов выбыли из исследования в связи с потерей контакта или по другим причинам, у 363 пациентов собирали данные через 1, 4 года и 10 лет, из них у 209 пациентов - ежегодно.

По материалам исследования создана открытая база данных (свидетельство Роспатента о государственной регистрации базы данных № 2014620990 от 10.07.2014), на основании которой выполнены расчеты, представленные в настоящей работе.

\section{Одобрение этического комитета}

Исследование одобрено комитетом по этике ФГБОУ ВО «Саратовский ГМУ им. В.И. Разумовского» Минздрава России.

\section{Обследуемая популяция}

В исследование включили пациентов с АС, соответствовавших модифицированным Нью-Йоркским критериям для АС [12], и пациентов с ПсА, соответствовавших критериям CASPAR (Classification Criteria of Psoriatic Arthritis, 2006) для ПсА [13]. Добровольцы без АС, ПсА и сердечно-сосудистых заболеваний составили группу сравнения.

Из 676 пациентов исследования ПРОГРЕСС, включенных исходно, 363 пациента наблюдались в центре в течение 10 лет, из них 238 - с диагнозом АС, $109-$ с диагнозом ПсА. Шестнадцать больных одновременно соответствовали модифицированным Нью-Йоркским критериям для АС (1984) [12] и критериям ПсА CASPAR [13], поэтому их данные анализировали отдельно, и в настоящей работе они не представлены.

Клиническая характеристика пациентов со СпА и здоровых лиц, включенных в анализ 10-летней сердечнососудистой заболеваемости, представлена в табл. 1.

Все пациенты с АС и ПсА за 10-летний период наблюдения принимали нестероидные противовоспалительные препараты (НПВП), индекс приема НПВП ASAS (Assessment of Spondyloarthritis International Society) для указанного периода интереса составил $40[20 ; 80] \%$, т. е. в среднем пациенты за 10 лет приняли $40 \%$ от максимально возможной для данного периода суммарной дозы. В группе АС 35 (14,1 \%) больных принимали метотрексат 7,5-25,0 мг/нед, 124 (52,1 \%) сульфасалазин 2,0-3,0 г/сут, 5 (2,1\%) - лефлуномид 20 мг/сут, 5 (2,1\%) - комбинированную терапию метотрексатом и сульфасалазином, 54 (22,8 \%) - глюкокортикоиды внутрь в дозе 7,5-10,0 мг/сут в преднизолоновом эквиваленте. В группе ПсА 35 (32,1 \%) больных принимали глюкокортикоиды внутрь по 7,5-10,0 мг/сут, 
Таблица 1. Клиническая характеристика пациентов со спондилоартритами и лии группы контроля, включенных в анализ 10-летней сердечнососудистой заболеваемости (данные на момент начала наблюдения)

\begin{tabular}{|c|c|c|c|c|}
\hline Показатель & $\begin{array}{c}\text { Спондилоартрит, } \\
n=363\end{array}$ & $\begin{array}{c}\text { Анкилозирующий } \\
\text { спондилит, } n=238\end{array}$ & $\begin{array}{l}\text { Псориатический } \\
\text { артрит, } n=109\end{array}$ & $\begin{array}{c}\text { Контрольная группа, } \\
n=150\end{array}$ \\
\hline Возраст, (M $\pm \mathrm{SD})$ лет & $40,1 \pm 14,1$ & $40,0 \pm 11,4$ & $40,5 \pm 10,6$ & $39,0 \pm 11,2$ \\
\hline Мужской пол, $n(\%)$ & $253(69,7)^{*, * *}$ & $212(76,25)^{*, * *}$ & $41(48,2)$ & $84(56)$ \\
\hline Длительность заболевания, (M \pm SD) лет & $13,9 \pm 11,2$ & $13,7 \pm 10,03$ & $14,8 \pm 14,4$ & - \\
\hline Возраст начала заболевания, (M \pm SD) лет & $27,1 \pm 11,0$ & $26,33 \pm 10,1$ & $29,5 \pm 13,1$ & - \\
\hline Возраст постановки диагноза, (M $\pm \mathrm{SD})$ лет & $33,9 \pm 11,5$ & $34,8 \pm 10,8$ & $33,4 \pm 13,6$ & - \\
\hline Артериальная гипертензия, $n$ (\%) & $56(15,4)$ & $32(11,5)^{* *}$ & $24(28,2)^{*}$ & $22(14,7)$ \\
\hline Курение, $n$ (\%) & $181(49,8)^{*}$ & $151(54,31)^{*, * *}$ & $30(35,2)$ & $40(26,7)$ \\
\hline
\end{tabular}

Примечание. «-»- отсутствие данных. *Статистически значимые различия при сравнении с распределением лии группы сравнения (точный критерий Фишера), $p<0,001 .{ }^{*}$ Статистически значимые различия при сравнении с составом больных псориатическим артритом, $p<0,001$.

По возрасту, длительности заболевания, возрасту начала заболевания, возрасту постановки диагноза показатели всех групп сопоставимы, $p>0,05$ для всех.

$75(68,8 \%)$ - метотрексат в дозе 7,5-25,0 мг/нед, 24 $(22,0 \%)$ - сульфасалазин 2,0-3,0 г/сут, 6 (5,5 \%) - лефлуномид 20 мг/сут, 5 (4,58 \%) - комбинированную терапию метотрексатом и сульфасалазином. Ингибиторы фактора некроза опухоли альфа (иФНО- $\alpha$ ) получали $39(16,4 \%)$ пациентов с АС и $11(10,9 \%)$ - с ПсА. Лекарственная терапия артериальной гипертензии указана в табл. 2.

В качестве лиц группы контроля в исследование ПРОГРЕСС были включены 182 здоровых добровольца, из них с 32 был потерян контакт, 150 человек продолжили наблюдение в течение 10 лет.

\section{Определение ищемической болезни сердца, оценка активности спондилоартритов и факторов сердечно-сосудистого риска}

Оценивали документально подтвержденные кардиологом случаи ишемической болезни сердца (ИБС) (стенокардия напряжения, острый коронарный синдром, нестабильная стенокардия, инфаркт миокарда).

Учитывали наличие факторов сердечно-сосудистого риска: возраст (55 лет и старше для мужчин и 60 лет и старше для женщин), мужской пол, семейный анамнез ранних сердечно-сосудистых событий, курение в настоящий момент и в анамнезе. Определяли и учитывали уровень общего холестерина сыворотки крови, липопротеидов низкой и высокой плотности, триглицеридов. Рассчитывали индекс массы тела как отношение массы тела в килограммах к возведенному в квадрат росту в метрах.

Для оценки активности болезни рассчитывали индексы активности BASDAI (Bath Ankylosing Spondylitis Disease Activity Index) [14], ASDAS (Ankylosing Spondylitis Disease Activity Score) [15], DAS 4 (Disease Activity Score) [16], скорость оседания эритроцитов (СОЭ), уровень С-реактивного белка (СРБ) (высокочувствительным методом, аппарат Hittachi).
Таблица 2. Антигипертензивная терапия пациентов с контролируемой артериальной гипертензией на момент включения в исследование, $n=56$

\begin{tabular}{|c|c|c|}
\hline Препараты & $\begin{array}{l}\text { Число пациентов, } \\
\text { получающих } \\
\text { препарат, } n\end{array}$ & $\%$ \\
\hline Диуретики & 16 & 28,57 \\
\hline $\begin{array}{l}\text { Ингибиторы ангиотензин- } \\
\text { превращающего фермента }\end{array}$ & 31 & 55,3 \\
\hline$\beta$-блокаторы & 22 & 39,28 \\
\hline Блокаторы кальциевых каналов & 6 & 10,71 \\
\hline $\begin{array}{l}\text { Ингибиторы ангиотензинпревра- } \\
\text { щающего фермента и } \beta \text {-блокаторы }\end{array}$ & 11 & 19,64 \\
\hline $\begin{array}{l}\text { Ингибиторы ангиотензинпревра- } \\
\text { щающего фермента и блокаторы } \\
\text { кальциевых каналов }\end{array}$ & 8 & 14,2 \\
\hline $\begin{array}{l}\text { Диуретики и ингибиторы ангио- } \\
\text { тензинпревращающего фермента }\end{array}$ & 26 & 46,42 \\
\hline
\end{tabular}

\section{Статистический анализ}

Статистический анализ выполнен с применением пакетов прикладных программ Statistica SPSS 17 и Statistica Graph Pad Prism. Характер распределения данных оценивали графическим методом и с использованием критериев Колмогорова-Смирнова и Шапиро-Уилка, нормальным считалось распределение при $p>0,05$. Описание признаков, имеющих нормальное распределение, представлено в виде $\mathrm{M} \pm \mathrm{SD}$, где $\mathrm{M}$ - среднее арифметическое, SD - стандартное отклонение; для признаков с распределением, отличным от нормального, результаты представлены в виде Me [Q1; Q3], где $\mathrm{Me}$ - медиана, Q1 и Q3 - 1-й и 3-й квартили. Для сравнения 2 групп с нормальным распределением количественного признака определяли $t$-критерий Стьюдента 
для независимых групп (с учетом вида дисперсии признака, определенного методом Левена), парный $t$-тест для зависимых групп. При характере распределения данных, отличном от нормального, применяли непараметрические методы: критерий Манна-Уитни, критерий Вальда-Вольфовица, критерий $\chi^{2}$, критерий Вилкоксона, критерий знаков. Сравнение частоты появления артериальной гипертензии изучали с применением метода Мантеля-Кокса (log-rank Mantel-Cox test). Рассчитывали отношение рисков (RR) развития артериальной гипертензии и отношение шансов для артериальной гипертензии для разных групп пациентов. Различия считались статистически значимыми при $p<0,05$ [17].

\section{Результаты}

За 10 лет наблюдения за больными СпА, не имевшими сердечно-сосудистых заболеваний на момент включения в исследование, новые случаи ИБС были зарегистрированы у 64 из 278 пациентов с АС и у 16 из 150 лиц группы сравнения, $p=0,0017$ (двусторонний точный критерий Фишера), и у 35 пациентов с ПсА $(n=85)$, что значимо превышало показатели лиц группы сравнения и показатели лиц с АC, $p<0,0001$ для попарного сравнения (двусторонний тест Фишера). Распределение новых случаев инфаркта миокарда и стенокардии напряжения у больных СпА и здоровых лиц представлено в табл. 3.

Таблица 3. Число новых случаев стенокардии напряжения и инфаркта миокарда у больных спондилоартритами и здоровых лии за 10 лет наблюдения (n (\%))

\begin{tabular}{|l|c|c|c|}
\hline Заболевание & $\begin{array}{c}\text { Анкилозирующий } \\
\text { спондилит, } \\
\boldsymbol{n}=\mathbf{2 7 8}\end{array}$ & $\begin{array}{c}\text { Псориатиче- } \\
\text { ский артрит, } \\
\boldsymbol{n = 8 5}\end{array}$ & $\begin{array}{c}\text { Контрольная } \\
\text { группа, } \boldsymbol{n = 1 5 0}\end{array}$ \\
\hline $\begin{array}{l}\text { Стенокардия } \\
\text { напряжения }\end{array}$ & $36(12,9)$ & $22(25,9)$ & $14(9,3)$ \\
\hline $\begin{array}{l}\text { Инфаркт } \\
\text { миокарда }\end{array}$ & $28(10,1)$ & $13(15,3)$ & $2(1,3)$ \\
\hline
\end{tabular}

Риск развития ИБС у больных ПсА превосходил таковой у лиц группы контроля: RR 4,16 (95 \% доверительный интервал (ДИ) 2,36-7,33) (рис. 1).

Частота появления стенокардии у больных ПсА превосходила частоту ее развития у лиц группы сравнения, $p=0,002$ (двусторонний тест Фишера). RR развития стенокардии у больных ПсА составил 2,65 (95\% ДИ 1,42-4,93), у пациентов с АС - 1,28 (95\% ДИ 0,9-2,3) (рис. 2).

Частота развития инфаркта миокарда у больных АС превосходила таковую у лиц группы контроля, $p=0,0015$ (двусторонний тест Фишера). У больных ПсА число новых случаев инфаркта миокарда превосходило число аналогичных случаев у лиц группы контроля, $p<0,0001$ (двусторонний тест Фишера).

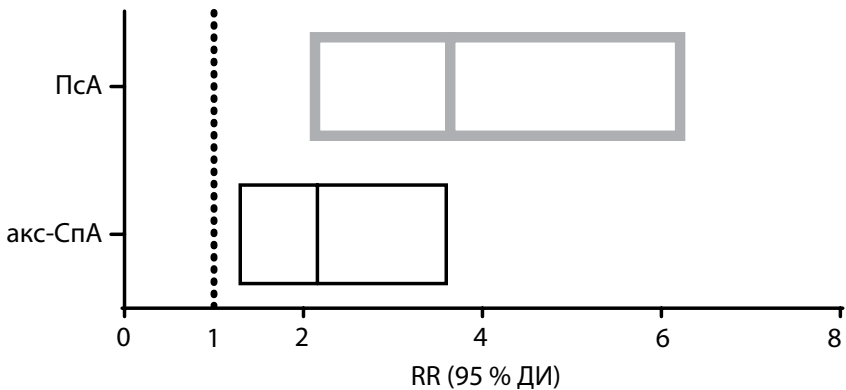

Рис. 1. Относительный риск (RR) развития ишемической болезни сердца у больных анкилозирующим спондилитом и псориатическим артритом. ДИ - доверительный интервал

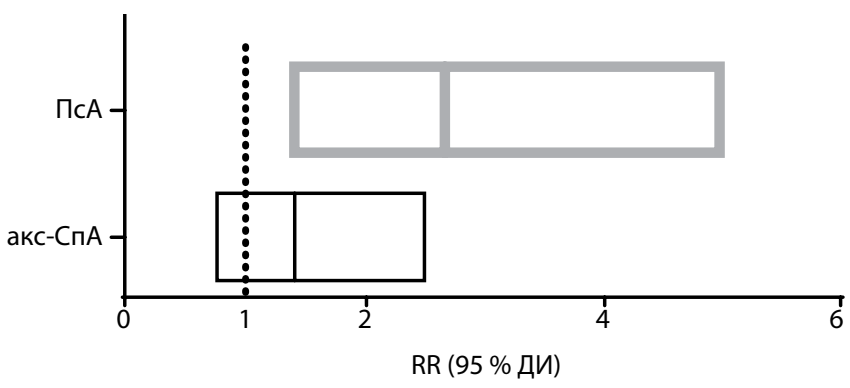

Рис. 2. Относительный риск (RR) развития стенокардии напряжения у больных анкилозирующим спондилитом и псориатическим артритом. ДИ - доверительный интервал

RR развития инфаркта миокарда относительно здоровых лиц был повышен как у больных AC (RR 4,98; $95 \%$ ДИ 1,54-6,12), так и у пациентов с ПсA (RR 5,2; $95 \%$ ДИ 2,4-7,8).

Так как в анализируемой нами выборке были случаи потери контакта как с пациентами, так и с лицами группы контроля, нельзя исключить возможность наличия патологических изменений, в том числе и тяжелых, именно среди лиц, с которыми потерян контакт. В связи с этим мы сочли необходимым выполнить сравнительный анализ встречаемости ИБС, стенокардии и инфаркта миокарда с ожидаемыми популяционными значениями и ожидаемыми значениями для больных АС (по данным европейских метаанализов) [22-26]. Сравнение частоты появления ИБС с применением log-rank Mantel-Cox test и log-rank test for trend показало различия между частотой появления ИБС у лиц без СпА, пациентов с АС и ПсА, $p<0,0001$. Ниже представлены кривые Каплана-Майера, демонстрирующие данные различия (рис. 3).

Установлена бо́льшая встречаемость инфаркта миокарда у больных АС по сравнению с ожидаемыми популяционными значениями $(6,4 \%), p=0,02$, и по сравнению с ожидаемой заболеваемостью инфарктом миокарда у больных АС в европейской популяции $(1,8 \%), p<0,001$.

\section{Обсуждение}

Настоящая работа показала повышение риска развития ИБС при СпА, продемонстрировав неоднородность заболеваемости ИБС при разных типах СпА. 


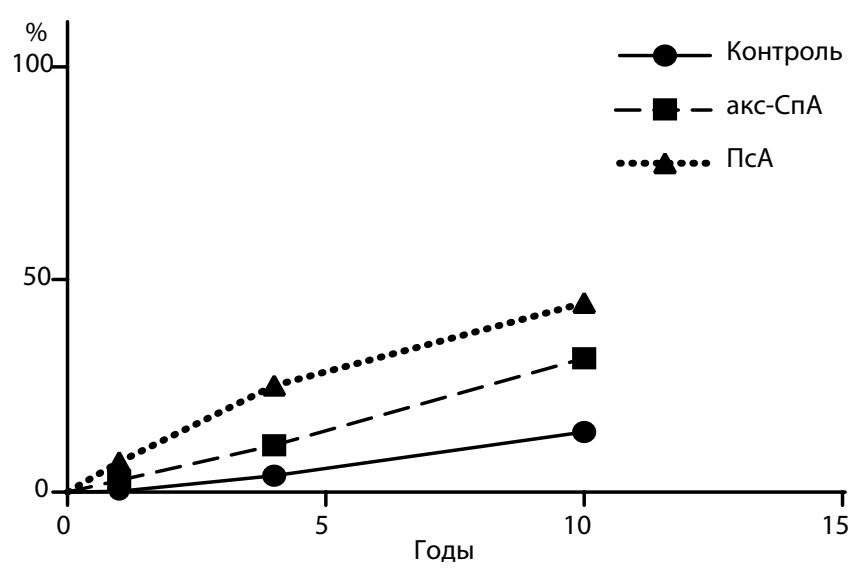

Рис. 3. Относительная частота (\%) появления новых случаев ишемической болезни сердиа у лиц, без спондилоартритов (контроль), лии с анкилозирующим спондилитом и больных псориатическим артритом, кривые Каплана-Майера

В целом полученные нами данные согласуются с работами, в которых отмечалось повышение сердечнососудистой заболеваемости при АС и ПсА, в частности с одним из первых исследований, показавшим повышение сердечно-сосудистой заболеваемости и смертности при СпА, - работой С. Нап и соавт. (2006) [2]. Немного позже (2010) M. Peters и соавт. показали, что наличие инфаркта миокарда у больных с АС наблюдается в 4,4\% случаев, в то время как в сопоставимой по факторам сердечно-сосудистого риска популяции лиц без артрита инфаркт миокарда был выявлен только в 1,2 \% случаев [20]. По данным шведского популяционного исследования [21], у больных АС $(n=935)$ установлено более частое, чем в популяции, развитие ИБС, артериальной гипертензии, сахарного диабета, атриовентрикулярных блокад. При наличии ИБС инфаркт миокарда чаще развивался у лиц с АC, чем без него [21]. В метаанализе, выполненном S. Mathieu и соавт. (2011), была показана тенденция к увеличению риска инфаркта миокарда при АС [21]. Статистической значимости различия с популяцией не достигли у лиц группы контроля $(n=82745)$ инфаркт миокарда встречался в 4,6 \% случаев, у пациентов с АС $(n=3279)-$ в 7,4 \% случаев. Метаанализ S. Mathieu и соавт. (2015) показал бо́льший риск возникновения инфаркта миокарда и инфаркта мозга у больных AC (RR 1,6; 95 \% ДИ 1,6-11,0 \%) [22, 23]. Трехкратное повышение сердечно-сосудистой заболеваемости при АC показано в исследовании K.A. Wright и соавт. (2015) и S.C. Heslinga и соавт. (2015) [5]. Интересно, что при дебюте AC в среднем возрасте повышение сердечно-сосудистого риска наблюдается через 5 лет от начала заболевания
[24]. Следует признать, что все перечисленные работы включали разнородные выборки пациентов, которым был установлен диагноз АС, в том числе при наличии псориаза и воспалительных заболеваний кишечника, что несколько уменьшает их значимость, так как данные выборки не могут трактоваться как выборки больных только с АС.

Если анализировать данные по сердечно-сосудистой заболеваемости и смертности при ПсА, то, как и при АС, отмечается постепенное увеличение количества работ, показывающих повышение сердечно-сосудистого риска у больных ПсА. D. Gladman и соавт. (1978, 1998) показали, что смертность при ПсА превышает популяционную на $65 \%$ у мужчин и на $59 \%$ у женщин, а преобладающей причиной смерти являются сердечно-сосудистые события. Противоречат указанным работам несколько исследований [25, 26], в том числе и работа S. Kondratiouk и соавт. (2008), в которой не выявлено повышения частоты атеротромбоза у больных ПсА [26]. Из факторов риска авторы отметили наличие у больных повышения артериального давления и индекса массы тела по сравнению с общей популяцией при отсутствии изменений уровня общего холестерина и глюкозы крови [26]. Не было установлено повышение риска смерти у пациентов с ПсА в популяции Великобритании (1985-2007): из 453 больных умерли 37 [25].

Выполненное нами исследование согласуется с вышеперечисленными работами - показано повышение риска развития ИБС у больных АС и ПсА без сердечно-сосудистых заболеваний. Преимуществами представленной нами работы явились проспективный характер наблюдения за пациентами, раздельный анализ сердечно-сосудистой заболеваемости для аксиальных форм спондилоартритов (АС) и периферических спондилоартритов (ПсА), что позволило установить повышение риска развития ИБС у больных ПсА по сравнению с АС за счет нефатальных инфарктов миокарда. Выявленные данные представляются важными для клинической практики, так как позволяют расценивать пациентов с АС и ПсА как лиц с повышенным риском развития ИБС.

\section{Заключение}

Риск развития стенокардии напряжения у больных AC не превышает аналогичный у лиц без СпА. При этом пациенты с АС имеют бо́льший риск развития ИБС и инфаркта миокарда, чем лица без СпА. Больные ПсА имеют бо́льший риск развития ИБС как по сравнению со здоровыми, так и с лицами, страдающими АС. 


\section{J И T}

1. Фоломеева О.М., Галушко Е.А., Эрдес Ш.Ф. Распространенность ревматических заболеваний в популяциях взрослого населения России и США. Научно-практическая ревматология 2008;(4):4-13. [Folomeeva O.M., Galushko E.A., Erdes Sh.F. The prevalence of rheumatic diseases in populations of the adult population of Russia and the United States. Nauchno-prakticheskaya revmatologiya $=$ Scientific and Practical Rheumatology 2008;(4):4-13. (In Russ.)]. 2. Han C., Robinson D.W. Jr., Hackett M.V. et al. Cardiovascular disease and risk factors in patients with rheumatoid arthritis, psoriatic arthritis, and ankylosing spondylitis. J Rheumatol 2006;33(11):2167-72.

3. Haroon N.N., Paterson J.M., Li P. et al. Patients with ankylosing spondylitis have increased cardiovascular and cerebrovascular mortality: a population-based study. Ann Intern Med 2015;163(6):409-16.

4. Ребров А.П., Гайдукова И.З., Поддубный Д.А. Кардиоваскулярная патология у больных анкилозирующим спондилитом. Научно-практическая ревматология 2012;(2):100-5. [Rebrov A.P., Gaydukova I.Z., Poddubnyy D.A. Cardiovascular disease in patients with ankylosing spondylitis. Nauchnoprakticheskaya revmatologiya $=$ Scientific and Practical Rheumatology 2012;(2):100-5. (In Russ.)].

5. Heslinqa S.C., Van den Oever I.A., Van Sijl A.M. et al. Cardiovascular risk management in patients with active ankylosing spondylitis: a detailed evaluation. BMC Musculoskelet Disord 2015;16:80. 6. Brophy S., Cooksey R., Atkinson M. et al. No increased rate of acute myocardial infarction or stroke among patients with ankylosing spondylitis - a retrospective cohort study using routine data. Semin Arthritis Rheum 2012;42(2):140-5.

7. Гайдукова И.3., Ребров А.П., Лебединская О.А. и др. Кардиоваскулярная заболеваемость и смертность при анкилозирующем спондилите и псориатическом артрите - результаты одноцентрового четырехлетнего наблюдения. Практическая медицина 2015;32(88):123-9. [Gaydukova I.Z., Rebrov A.P., Lebedinskaya O.A. et al. Cardiovascular morbidity and mortality in ankylosing spondylitis and psoriatic arthritis - the results of a single-site four-year follow-up. Prakticheskaya meditsina $=$ Practical Medicine 2015;3-2(88):123-9. (In Russ.)]. 8. Sundström B., Johansson G., Johansson I., Wållberg-Jonsson S. Modifiable cardiovascular risk factors in patients with ankylosing spondylitis. Clin Rheumatol 2014;33(1):111-7.

9. Berg I.J., Semb A.G., van der Heijde D. et al. Uveitis is associated with hypertension and atherosclerosis in patients with ankylosing spondylitis: a cross-sectional study. Semin Arthritis Rheum 2014;44(3):309-13. 10. Поддубный Д.А., Ребров А.П. Традиционные и новые факторы риска развития сердечно-сосудистых заболеваний у больных анкилозирующим спондилитом (болезнь Бехтерева). Терапевтический архив 2007;79(5):20-4. [Poddubnyy D.A., Rebrov A.P.

Traditional and new risk factors for cardiovascular disease in patients with ankylosing spondylitis (ankylosing spondylitis). Terapevticheskiy arkhiv $=$ Therapeutic

Archive 2007;79(5):20-4. (In Russ.)]. 11. Brooks W.B., Jordan J.S., Divine G.W. et al. The impact of psychologic factors on measurement of functional status. Assessment of the sickness impact profile. Med Care 1990;28(9):793-804.

12. Van der Linden S., Valkenburg H.A., Cats A. Evaluation of diagnostic criteria for ankylosing spondylitis. A proposal for modification of the New York criteria. Arthritis Rheum 1984;27(4):361-8. 13. Taylor W., Gladman D., Helliwell P. et al. Classification criteria for psoriatic arthritis: development of new criteria from a large international study. Arthritis Rheum 2006;54(8):2665-73.

14. Garrett S., Jenkinson T., Kennedy L.G. et al. A new approach to defining disease status in ankylosing spondylitis: the Bath Ankylosing Spondylitis Disease Activity Index. J Rheumatol 1994;21(12):2286-91. 15. Lukas C., Landewé R., Sieper J. et al. Development of an ASASendorsed disease activity score (ASDAS) in patients with ankylosing spondylitis. Ann Rheum Dis 2009;68(1):18-24.

16. Wells G., Becker J.C., Teng J. et al. Validation of the 28-joint Disease Activity Score (DAS28) and European League Against Rheumatism response criteria based on C-reactive protein against disease progression in patients with rheumatoid arthritis, and comparison with the DAS28 based on erythrocyte sedimentation rate. Ann Rheum Dis 2009;68(6):954-60.

17. Реброва О.Ю. Статистический анализ медицинских данных. М.: Медиа Сфера, 2002. [Rebrova O.Yu. Statistical analysis of medical data. Moscow: MediaSfera, 2002. (In Russ.)].

18. Heredia E., Zhu B., Lefevre C. et al. Prevalence and incidence rates

of cardiovascular, autoimmune, and other diseases in patients with psoriatic or psoriatic arthritis: a retrospective study using Clinical Practice Research Datalink. Edson- J Eur Acad Dermatol Venereol 2015;29(5): 955-63.

19. Favarato M.H., Mease P., Gonçalves C.R. et al. Hypertension and diabetes significantly enhance the risk of cardiovascular disease in patients with psoriatic arthritis. Clin Exp Rheumatol 2014;32(2):182-7.

20. Peters M.J., van der Horst-Bruinsma I.E., Dijkmans B.A., Nurmohamed M.T.

Cardiovascular risk profile of patients with spondyloarthropathies, particularly ankylosing spondylitis and psoriatic arthritis. Arthritis Rheum 2004;34(3):585-92.

21. Mathieu S., Gossec L., Dougados M., Soubrier M. Cardiovascular profile in ankylosing spondylitis: a systematic review and meta-analysis. Arthritis Care Res (Hoboken) 2011;63(4):557-63.

22. Mathieu S., Soubrier M. Cardiovascular risk in ankylosing spondylitis. Presse Med 2015;44(9):907-11.

23. Mathieu S., Pereira B., Soubrier M. Cardiovascular events in ankylosing spondylitis: an updated meta-analysis. Semin Arthritis Rheum 2015;44(5):551-5.

24. Hung Y.M., Chang M.P., Wei J.C. et al. Midlife Ankylosing Spondylitis Increases the Risk of Cardiovascular Diseases in Males 5 Years Later: A National Population-Based Study. Medicine (Baltimore)

2016;95(18):e3596.

25. Buckley C., Cavill C., Taylor G. et al. Mortality in psoriatic arthritis - asinglecenter Study from the UK. J Rheumatol 2010;37(10):2141-4.

26. Kondratiouk S., Udaltsova M., Klatsky A.L. Associations of psoriatic arthritis and cardiovascular conditions in a large population. Perm J 2008;12(4):4-8. 